\title{
Validation of a Novel Algorithm for Ventricular Repolarization Analysis: Use of Physionet Resources
}

\author{
F Cantini, M Emdin, C Passino, M Varanini, F Conforti \\ CNR Institute of Clinical Physiology, Pisa, Italy
}

\begin{abstract}
Ventricular repolarization analysis allows extraction from the ECG signal of quantitative indexes (namely the QT interval), of prognostic value in unselected populations and cardiac patients, being related with arrhythmic risk. Several attempts to improve automatic ECG waveform detection have been accomplished, using signal derivatives, digital filtering, wavelet analysis, neural network techniques, nonlinear approaches. In the present study, a single-lead low-pass differentiation detector of ECG significant points (PulseMeter) has been evaluated. The algorithm performance has been validated against the manual annotation of the "QT database" (http://www.physionet.org/), developed for validation purposes. QRS complex and other ECG waveform boundaries were independently evaluated in the present study. The mean values and standard deviations computed improve the result of automatic annotation in $Q T$ database, especially in $T$ wave detection. The QRS detector has a sensitivity of $99.96 \%$ and a positive predictivity of $99.96 \%$ on the first lead and a sensitivity of $99.90 \%$ and a positive predictivity of 99.94\% on the second lead, showing a better performance than the automatic annotation in the $Q T$ database.
\end{abstract}

\section{Introduction}

The analysis of ventricular repolarization allows to compute quantitative indexes (such as JT, QT interval) with diagnostic and prognostic values in patients with systemic (i.e. diabetes) or cardiovascular diseases, because of their linkage with arrhythmias or sudden death.

The development of automatic algorithms for ventricular repolarization analysis from the electrocardiographic signal is relevant to the diagnostic process in subsets of patients with potential arrhythmic or ischemic risk, either during provocative tests, ambulatory or Intensive Care Unit ECG monitoring.
In the present study, a single-lead, low-pass differentiation detector of ECG significant points (PulseMeter) (figure 1) has been evaluated.

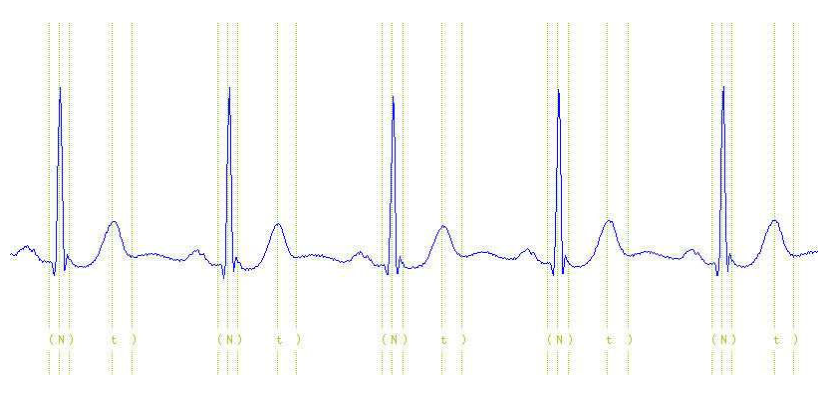

Figure 1: Application of PulseMeter to the ECG signal: automatic annotation of significant points

\section{Methods}

The "Physionet QT database" [1] has been used for PulseMeter algorithm performance evaluation. The database is a collection of 15-minute long, 2-lead, 105 selected ECG recordings from other databases such as MIT-BIH Arrhythmia Database [7], European ST-T Database [8] and Boston Beth Israel Deaconess Medical Center database.

Signals have been selected to cover a wide range of QRS, ST segment and T wave morphologies. For each record a set of annotation for the QRS complex and a set of manual annotations for $\mathrm{T}, \mathrm{U}$ and $\mathrm{P}$ wave boundaries for selected beats are provided. For 11 records a second set of manual annotations is provided. Also, automatic annotations obtained using the "ecgpuwave" application are available [5][6].

\subsection{Preprocessing}

While processing biological signals [2], filter cut-off characteristics are not critical in order to extract information from the signals. Thus, for our purpose, moving-average digital filters were used. This kind of 
FIR filters have some advantages: moving-average filters are incremental and can be implemented for any sampling frequency.

A low-pass filter has been implemented, using multiple moving-average filters with different length in order to improve the cut-off characteristics of the single moving-average filter.

Figure 2 show the frequency response of a filter, implemented with double moving-average.

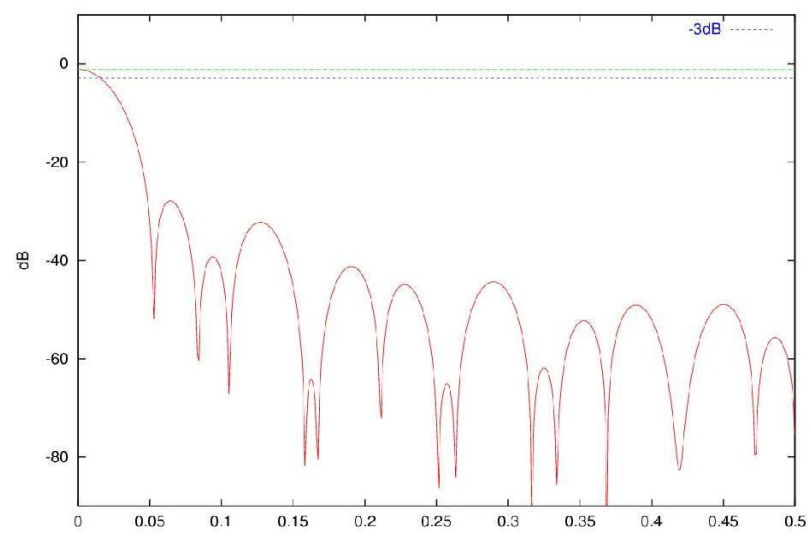

Figure 2: Double low-pass moving-average filter frequency response

The high-pass filtered signal is obtained as a difference between the original and a low-pass filtered signal.

\subsection{Waveform detection}

For each lead, QRS complex, QRS onset and offset, T wave peak and offset were detected independently. First QRS complexes were detected, then QRS boundaries were determinated. Finally, $T$ wave peak and offset detection were performed between two consecutive QRS complexes.

Detection of the QRS complex and QRS boundaries was based on low-pass and high-pass moving-average filtering, signal differentiation and local maximum or minimum search respectively. For the QRS complex detection, a $\mathrm{T}$ wave mask was used in order to reduce false positive detections.

Detection of $\mathrm{T}$ wave was also based on movingaverage filtering, signal differentiation and local maximum search.

\section{Validation}

In order to validate the algorithm, first the QRS complex detector has been evaluated, thereafter the waveform boundaries (QRS and $\mathrm{T}$ wave). Automatic annotations from either "PulseMeter" or "ecgpuwave" have been compared using as reference manual annotations. Comparisons between two sets of manual annotations have been performed in order to evaluate the inter-observer variability between different expert annotators.

QRS complex detector has been validated by computing parameters such as Sensitivity (S) and Positive Predictivity (PP):

$$
S=\frac{n}{(n+f(-))} \quad P P=\frac{n}{(n+f(+))}
$$

where $n$ are the right detections, $f(+)$ is the number of automatic annotation that are not recorded in the set of manual annotations (false positives) and $f(-)$ is the number of manual annotations that are not recorded in the automatic annotation set (missed beats). Annotations in files record.atr have been used as reference. S and PP were calculated using $b x b$ application [7] with a $150 \mathrm{~ms}$ match window (default value for $b x b$ ) and excluding the first and the last 10 second periods for each record.

As concerns the waveform boundaries detector validation, the differences between manual and automatic annotation were calculated, both for "PulseMeter" and "ecgpuwave", for R wave, QRS onset and offset, $\mathrm{T}$ wave peak and offset, then the mean value and the SD of the difference were calculated.

As concerns the QT interval measures, linear regression has been performed and R-square parameter has been calculated, where

$$
R^{2}=\frac{S S_{\text {regression }}}{S S_{\text {total }}}
$$

and SS is the sum of squares.

Both "PulseMeter" and "ecgpuwave" are single channel detectors and generate annotations for each lead of the record.

Since the manual annotations on QT database were made by experts using two leads, automatic annotations closer to the manual ones have been used to perform the comparison for each significant point.

\section{Results}

\subsection{Comparison between manual annotations}

In 11 records a second, independent set of manual annotations has been used in order to evaluate the inter- 
observer variability between different expert annotators. Mean value and SD were calculated for each $\mathrm{R}$ wave, QRS onset and offset, $\mathrm{T}$ wave peak and offset measures, as shown below in table 1 .

Table 1

\begin{tabular}{lcc}
\hline & Mean $(m s)$ & $S D(m s)$ \\
\hline R wave & 0 & 2 \\
QRS onset & -4 & 14 \\
QRS offset & -3 & 17 \\
T peak & -4 & 30 \\
T offset & 6 & 40 \\
QT interval & 2 & 43 \\
\hline
\end{tabular}

A scatterplot of QT interval measures calculated using the first manual annotation set versus the corresponding value using the second set is reported below.

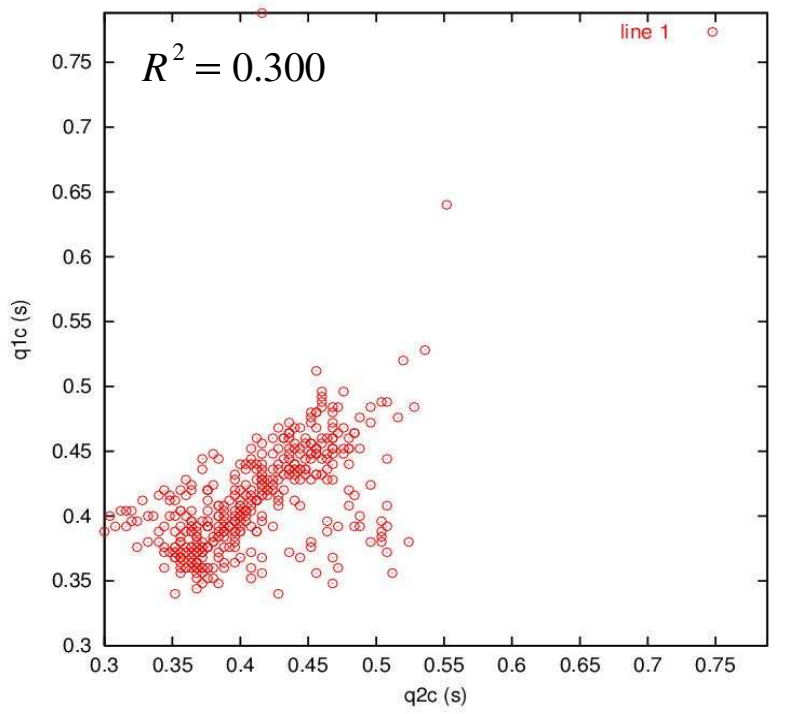

Figure 3: QT interval (manual set 1 vs manual set 2)

\subsection{Comparison between manual annotations and "ecgpuwave" annotations}

Automatic annotations provided with QT database were compared with manual reference annotations. $\mathrm{S}$ and PP are reported for each lead (table 2).

Table 2

\begin{tabular}{ccc}
\hline Lead & Sensitivity & $P P$ \\
\hline 0 & $99.87 \%$ & $99.86 \%$ \\
1 & $99.86 \%$ & $99.55 \%$ \\
\hline
\end{tabular}

Mean values and SD are reported for the each ECG measure in the table below.

Table 3

\begin{tabular}{lcc}
\hline & Mean $(m s)$ & $S D(m s)$ \\
\hline R wave & 9 & 17 \\
QRS onset & 3 & 11 \\
QRS offset & 0 & 10 \\
T peak & 6 & 31 \\
T offset & 12 & 55 \\
QT interval & -16 & 57 \\
\hline
\end{tabular}

A scatterplot of QT interval calculated using "ecgpuwave" annotation set versus the corresponding value using the first manual set is reported below.

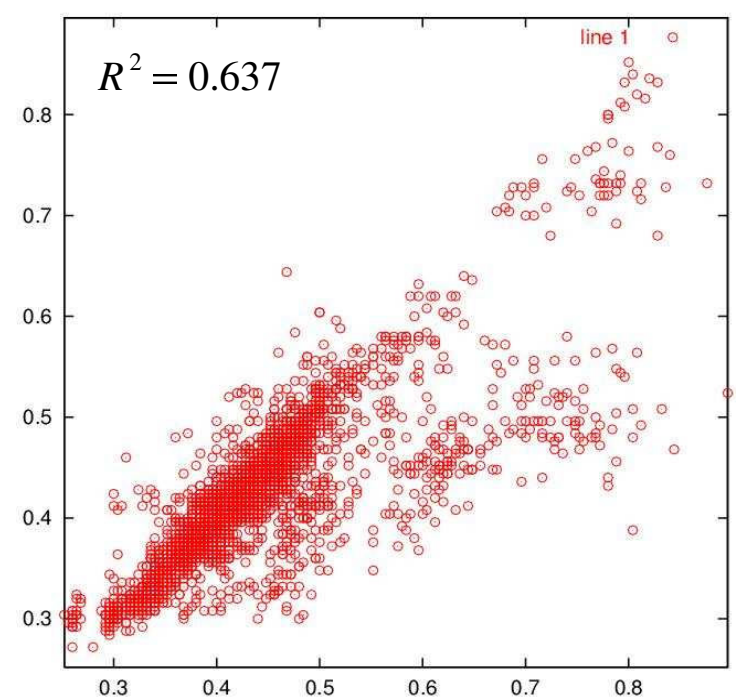

Figure 4: QT interval (ecgpuwave vs manual set)

\subsection{Comparison between manual annotations and "PulseMeter" annotations}

Automatic annotations made by our algorithm were compared with manual reference annotations. $\mathrm{S}$ and PP are reported for each lead (table 4).

Table 4

\begin{tabular}{ccc}
\hline Lead & Sensitivity & $P P$ \\
\hline 0 & $99.96 \%$ & $99.96 \%$ \\
1 & $99.90 \%$ & $99.94 \%$ \\
\hline
\end{tabular}


Mean values and SD are reported below for each ECG measure.

Table 5

\begin{tabular}{lcc}
\hline & Mean $(m s)$ & $S D(m s)$ \\
\hline R wave & -9 & 17 \\
QRS onset & 7 & 15 \\
QRS offset & 2 & 20 \\
T peak & -6 & 29 \\
T offset & 13 & 37 \\
QT interval & -6 & 38 \\
\hline
\end{tabular}

A scatterplot of QT intervals calculated using "PulseMeter" annotation set versus the corresponding value using the first manual set is reported.

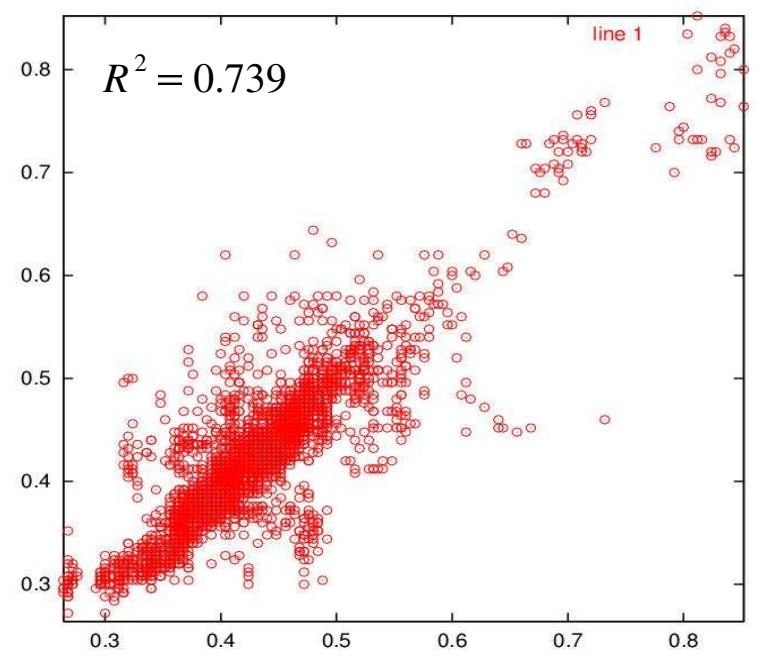

Figure 5: QT interval (PulseMeter vs manual set)

It is worthwhile noticing that there is a significant improvement in comparison with "ecgpuwave" results.

\section{Conclusions}

A single-lead, beat-by-beat, low-pass differentiation detector of ECG significant points, using movingaverage filters for the study of ventricular repolarization has been developed.

The comparison between two sets of independent manual annotations made by experts shows, as concerns the QT interval, a SD of $43 \mathrm{~ms}$, about $10 \%$ of the mean value of the interval.
The same kind of analysis made using the ecgpuwave algorithm and manual annotations shows a variability comparable to the inter-observer variability for all significant points except for the $\mathrm{R}$ wave.

The evaluation of PulseMeter shows results comparable to the inter-observer variability with a significant improvement for $\mathrm{T}$ offset and QT interval, as compared with the "ecgpuwave" annotation.

\section{References}

[1] Laguna P, Mark RG, Goldberg A, Moody GB. A database for evaluation of algorithms for measurement of QT and other waveform intervals in the ECG. Proceedings of Computers in Cardiology, Cambridge, Massachusetts. 1997;24:673-676.

[2] Thakor N, Webster JG, Tompkins WJ. Estimation of QRS complex power spectra for design of a QRS filter. IEEE TBE 1984;BME-31:702-705.

[3] Langley P, Murray A. Comparison of manual and automatic QT dispersion measurements in clinical groups. Proceedings of Computers in Cardiology, Cambridge, Massachusetts. 2001;28:645-648.

[4] Laguna P, Janè R, Caminal P. Automatic detection of wave boundaries in multilead ECG signals: validation with the CSE database. Comp Biom Res. 1994;27:45-60.

[5] Janè R, Blasi A, Garcia J, Laguna P. Evaluation of an automatic threshold based detector of waveform limits in holter ECG with the QT database. Proceeding of Computers in Cardiology Cambridge, Massachusetts. 1997;24:295-298.

[6] Laguna P, Vigo D, Janè R, Caminal P. Automatic wave onset and offset determination in ECG signals: validation with CSE database. Proceedings of Computers in Cardiology, Cambridge, Massachusetts 1992;19:167-170.

[7] Moody GB, Mark RG. The MIT-BIH arrhythmia database on CD-ROM and software for use with it. Proceedings of Computers in Cardiology, Cambridge, Massachusetts. 1990; 17:185-188

[8] Taddei A, Biagini A, Distante G, Emdin M, Mazzei MG, Pisani P, Roggero N, Varanini M, Mark RG, Moody GB, Braaksma L, Zeelenberg C, Marchesi C. The European ST-T database: development, distribution and use. Proceedings of Computers in Cardiology, Cambridge, Massachusetts. 1991;18:177-180.

Address for correspondence:

Federico Cantini, BS

CNR Institute of Clinical Physiology

Via G. Moruzzi, 1

56100 Pisa, Italy

cantini@ifc.cnr.it 